\title{
Lean Burn Natural Gas Engine R\&D
}

\section{CRADA 94-0260 with Cummins, Inc.}

\begin{abstract}
.
The primary objective of this cooperative research is to develop and verify models of internal combustion engine spark ignition devices in order to improve combustion chamber fuel ignition characteristics and to improve spark plug durability. As a direct result of this joint research, a novel spark plug design was improved. A theory of spark arc motion was developed that explains experimentally observed effects not explained by other published theories. The knowledge developed by this research will be used to further improve spark plugs as well as improve the ignition process in a combustion chamber.
\end{abstract}

The predictive models developed here are compared with experimental measurements, including highspeed photographs, of the spark as it translates across the gap. Two different spark plug configurations were investigated: the conventional or J-gap plug, and a novel spark ignition device (the FANG plug) invented by Cummins, Inc., the CRADA partner.

A description of the physics of arc dynamic motion in a spark plug gap, including the effects of an imposed transverse magnetic field, appears here in Appendix A as a result of the analytical effort. The theory proposed here does explain experimentally observed effects not completely explained by other research publications appearing in the scientific literature. These effects are due to pressure and ion, electron, and electrode interactions. A dominant mechanism for electrode erosion is presented for both spark plug configurations. Reversing the polarity of both types of spark plugs has verified this proposed erosion mechanism, according to data collected at Cummins.

An extensive series of experiments measured the arc position, voltage, and current as a function of time during the approximately 2 millisecond spark discharge. FANG plug data, obtained with the fast-framing camera experimental apparatus operating at 200,000 frames per second, are presented that show the transverse arc velocity varying directly as the inverse square root of the elapsed time since arc initiation. At the request of Cummins, experiments were performed on three conventional spark plugs identical in design and having the same spark gap, but differing as follows: one was new, another had been used in an engine, and the third was new but had been sandblasted to simulate a used plug. Cummins had observed that only the used plug required a significantly higher breakdown voltage. Experiments at ORNL indicated that the used plug had a significantly higher breakdown voltage confirming the Cummins observations (although the sandblasted plug also exhibited a higher breakdown voltage than the new plug but lower than the used plug), and thus an apparent increase of the arc breakdown voltage results as the plug ages in use. Further analysis of this phenomenon is warranted.

\section{Introduction.}

This CRADA coupled the analytical and experimental expertise of Oak Ridge National Laboratory with the heavy-duty internal combustion engine expertise of Cummins, Inc., of Columbus, Indiana, to investigate an innovative spark ignition device for natural gas fuelled internal combustion engines. This 
device is disclosed in U.S. Patent Numbers 5,555,862 and 5,619,959, invented by Luigi Tozzi of Cummins. Cummins has referred to this device as the Field Assisted Natural Gas, or "FANG", plug.

The FANG plug has a central electrode and a ground electrode having a partially slanted portion with respect to the central electrode. A permanent magnet placed inside the shell imposes a transverse magnetic force upon the spark arc such that arc moves along the slant electrode and thus elongates during the discharge time period. The FANG spark ignition device ("spark plug") had two configurations during this CRADA —old, and new. Cummins had modified the design configuration during the reporting period. Cummins also supplied two "half" plugs of both the old and new configuration, where half of the threaded portion of the spark plug shell was removed for experimental visualization of the arc.

The ORNL effort consists of two components: analytical and experimental. The analytical effort was based on the expertise of John $\mathrm{H}$. Whealton, who has considerable experience in the prediction of plasma performance as evidenced by his many publications in this area, and James C. Conklin, whose expertise is in heat transfer and thermal sciences. Isidor Sauers, who has the laboratory skills and extensive experience necessary to measure the performance of high voltage systems, performed the experimental activities.

This report documents the activities during 1999 and 2000 when James C. Conklin was the principal investigator for this CRADA.

\section{Analytical Activities.}

\section{Spark arc dynamics.}

A draft description of the physics of arc dynamic motion in a transverse magnetic field as applicable to the FANG plug was written by J. H. Whealton and is presented in Appendix A. This description includes the effects of ion, electron, and electrode interaction throughout the path length and ends of the arc as a function of time. This description specifically suggests a dominant mechanism for electrode erosion applicable for both the FANG plugs and conventional plug designs. The conventional analysis of arc motion in a magnetic field does not explain pressure dependence, especially at low pressure. The proposed theory here does indeed explain experimentally observed effects at various pressures as discussed during technical information exchange at Cummins.

\section{Electrode Erosion.}

At the request of Cummins, an estimate was made for the electrode material erosion rate due to electron bombardment during the breakdown (avalanche) phase of the spark event. A first estimate for an upper bound predicted excessive erosion rates as compared to those observed by Cummins. Further physical mechanisms were refined to better estimate erosion rates.

Because the ground electrode is at a positive potential with respect to the center electrode, the electrons are proposed as responsible for the erosion, presumably by local vaporization since sputtering-like phenomena are not likely due to a dearth of momentum. The likely occurrence of this erosion is at the end of the breakdown phase where a burst of electrons strikes a small area on the anode (slanted and ground electrode). This electron outburst occurs at relatively high energy of at least several electron volts and high current but for only a nanosecond. Accordingly, the electron beam vaporizes a little of the anode electrode. On the next spark event, the electron beam vaporizes a spot next to the first one. Finally the erosion is like an electron beam milling machine, rastering up the unslanted section of the anode and then 
starting up from the bottom again, repeating the process. Eventually, the unslanted part of the anode results in an eroded shiny mirror-like surface.

This mechanism explains why the anode electrode in the FANG plug becomes significantly more eroded than the cathode, and why the slanted part of the FANG gets much less erosion. For a conventional spark plug, the cathode also gets eroded because of positive ion impact, which occurs in the same place event after event. For the FANG plug, positive-ion erosion is diluted over a much larger area due to the arc movement in the magnetic field. The slanted part of the anode erodes much less compared to the straight part, because the end of the breakdown phase, where the erosion occurs, only happens at the beginning of the discharge, which in turn only happens at the straight part of the anode.

\section{Electrode Polarity Effects.}

Also at the request of Cummins, the effect of magnetic and electric field reversal was analyzed theoretically with respect to cathode and anode spot motion and erosion. The predicted arc motion and electrode erosion due to magnetic and electrode polarity reversal were in accord with experimental observations by Cummins.

Reversing the polarity of the spark plug electrodes can make a laboratory test of this mechanism. If the proposed mechanism is dominant, then the center electrode will be eroded only at the part opposite the flat part of the slanted electrode. If this is so, it can be argued that most other erosion mechanisms are less important than the proposed one (because other things would happen). If the proposed erosion mechanism is dominant, a reduction in the erosion rate can be obtained by reducing the peak current during the end of the breakdown phase. Exactly how to realize this limited current will be evaluated upon confirmation of this proposed erosion mechanism.

\section{Experimental Activities.}

\section{Experimental Apparatus.}

In order to quantify the spark arc position, voltage, and current as a function of time during the discharge, the following experimental configuration was developed. A sketch is presented in Figure 1, and a photograph of the experiment is given in Figure 2. Cummins provided the control box, coil, cell, and spark plug(s). The camera is a fast-framing Imacon 790, operating at 200,000 frames per second. A schematic drawing of the photographic record from the camera is presented in Figure 3, showing ten frames of five microsecond intervals and one microsecond exposure time per frame during a representative spark event. A photograph of a typical event, as recorded by the camera, is given in Figure 4.

\section{Cummins FANG plug experiments.}

As described in the introduction, the FANG plug incorporates a permanent magnet so that the arc moves along the slanted ground electrode during the discharge. The FANG plug had two configurations that we tested during this CRADA - referred to in this report as "old" and "new". Cummins had modified the design configuration during the reporting period. The new design incorporates some design improvements that were discussed between ORNL and Cummins personnel during a meeting in Columbus, Indiana, in 1999. Cummins also supplied two "half" plugs of both the old and new configuration, where half of the threaded portion of the spark plug shell was removed for experimental visualization of the arc. 
Cummins also supplied the different ignition systems for both plug designs. The new design requires less current than the old design. The triggering scheme for the new design, however, required modifications to obtain images similar to the ones obtained with the previous old design configuration. These triggering modifications were delayed due to camera problems. The problems were resolved and arcs of the new FANG plug were imaged successfully.

\section{Old FANG design.}

We have taken numerous images of the arc in an older design FANG plug in two configurations, one with half the magnets. The images, as shown on Figure 5, have a time dependence of the transverse arc velocity that decreases approximately linearly as the inverse square root of time from arc initiation. This inverse square root time dependence appears for both levels of magnetic field strength, but the velocity of the higher magnetic field strength (full FANG plug) is approximately twice that of the lower magnetic field strength plug (half FANG plug). Interestingly, the arc movement at the anode (ground electrode) of the lower magnetic strength half-plug is delayed for about 200 microseconds after breakdown as compared to the immediate arc movement on the cathode for both magnetic field strength plugs and the anode of the higher strength plug. This unexpected delay can be explained by the proposed theory of arc motion presented in Appendix A. These arc movement observations may have an important implication for electrode erosion.

\section{New FANG design.}

The new FANG half plug and its associated electronics were received during the second quarter of 2000. This version of the FANG is similar to the previous version in its basic electrode geometry but with different dimensions particularly with respect to the distances between the electrodes and the dielectric. The plug that we received had a very small minimum electrode gap spacing that was not in accordance with the specifications. Cummins has regapped the plug and experiments will be performed as funding permits.

Cummins is very interested in the arc velocity of the new FANG plug powered by their low current spark ignition coil. Previously we have been generating sparks with the high current ignition system with a peak current (in the glow phase) of 0.6 ampere. In comparison the low current source is 0.1 ampere peak current in the glow phase (not to be confused with the peak nanosecond scale breakdown current which is much higher). While a pulser that we controlled triggered the old high current system externally, the low current system had an internal pulser that created problems in triggering the camera.

Modifications to the low current system have been made which now allows proper triggering of the camera and external light source. Initial images of the new FANG plug arc produced by the low current system indicate that the spark is too weak to image with the present setup. Several options have been proposed to overcome this problem for future work, including (1) using a slower (lower framing speed) plug-in framing module with a longer exposure time, (2) modification of the presently used module to increase the exposure time, (3) and increasing the spark chamber gas pressure to increase the spark energy and spark luminosity. The first option of a slower framing module is the most expedient if a new module is readily available.

Images were taken of the full FANG plug that showed the arc movement. These data indicate that the arc movement of the new FANG plug is similar to the old FANG plug. Repeated measurements have been performed to determine the fraction of arcs that move laterally, i.e., move towards the dielectric instead of towards the end of the plug. 


\section{Conventional spark plug studies.}

An informal meeting was held at Cummins on June 8, 2000 to discuss the following topics: (1) progress on the FANG imaging and erosion modeling, and (2) issues on spark plug wear of conventional J-gap plugs. The first topic is reported in the above paragraphs. The second topic is a new development that Cummins personnel believe ORNL expertise on spark breakdown may help explain. Cummins has observed that the voltage required to initiate spark breakdown across the gap of a worn plug is increased significantly (by several kilovolts) over that for a new plug. This aging phenomenon could not be attributed solely to an increase of the gap due to electrode wear. An increase in electrode separation was not sufficient to account for the large increase in measured breakdown voltage. One proposed hypothesis was that leakage currents in a worn spark plug could produce a voltage drop across components preceding the spark gap, such as the ignition wire and spark plug internal resistance. If leakage currents do occur, the voltage produced at the coil secondary must be higher in order to initiate arc breakdown across the spark plug gap.

A DC discharge circuit has been implemented to provide a slowly increasing voltage that can be monitored, having a ramp time of about six seconds. Voltage probes were placed at both ends of a 9000 ohm standard ignition wire to determine if a voltage drop, which would be due leakage currents, exists prior to breakdown. Experiments on the three plugs at high pressure (9 bar) had begun immediately prior to the expiration of this CRADA.

We have examined three J-gap (conventional) spark plugs from Cummins to determine why spark breakdown voltages are significantly higher in a used plug than in a new plug with the same spark gap. The three plugs included (1) a used worn plug that was taken from an engine after several hundred hours of operation, (2) a new spark plug of the same spark gap geometry, and (3) a new plug that had been sandblasted to simulate the worn electrode surface of a used plug. While these tests have not been completed we have been able to run the following experiments. All three plugs were subjected to breakdowns using either a spark ignition coil (the old high current Cummins coil that we used for the earlier FANG studies) or a direct current source where the high voltage was slowly ramped over several seconds until breakdown. All the studies were done at both atmospheric pressure and at higher pressure of 200 psig. The objective was to observe pre-breakdown current that might account for a large voltage drop across the ignition wire, leading to a higher "effective" voltage required to breakdown the gap. So far we have not observed any significant current to account for the several kilovolt difference observed between the breakdown of the used plug and that of a new plug having the same gap spacing.

We also observed that the breakdown voltage of the new sandblasted plug fell between the values observed for the new non-sandblasted and the used plugs. This suggests that effect of the electrode surface condition on the breakdown voltage cannot be ruled out as the cause of the increased breakdown voltage of the used plugs having the same gap. Another explanation for the increase in apparent breakdown voltage of the used plug may be due to internal leakage, leading to higher breakdown voltages than would be expected based on the external electrode gap spacing. There is general agreement with Cummins, however, that the used plug has a significantly increased breakdown voltage than a new or new sandblasted plug having identical electrode gap spacing. Additional studies are warranted to determine the cause of this phenomenon. 


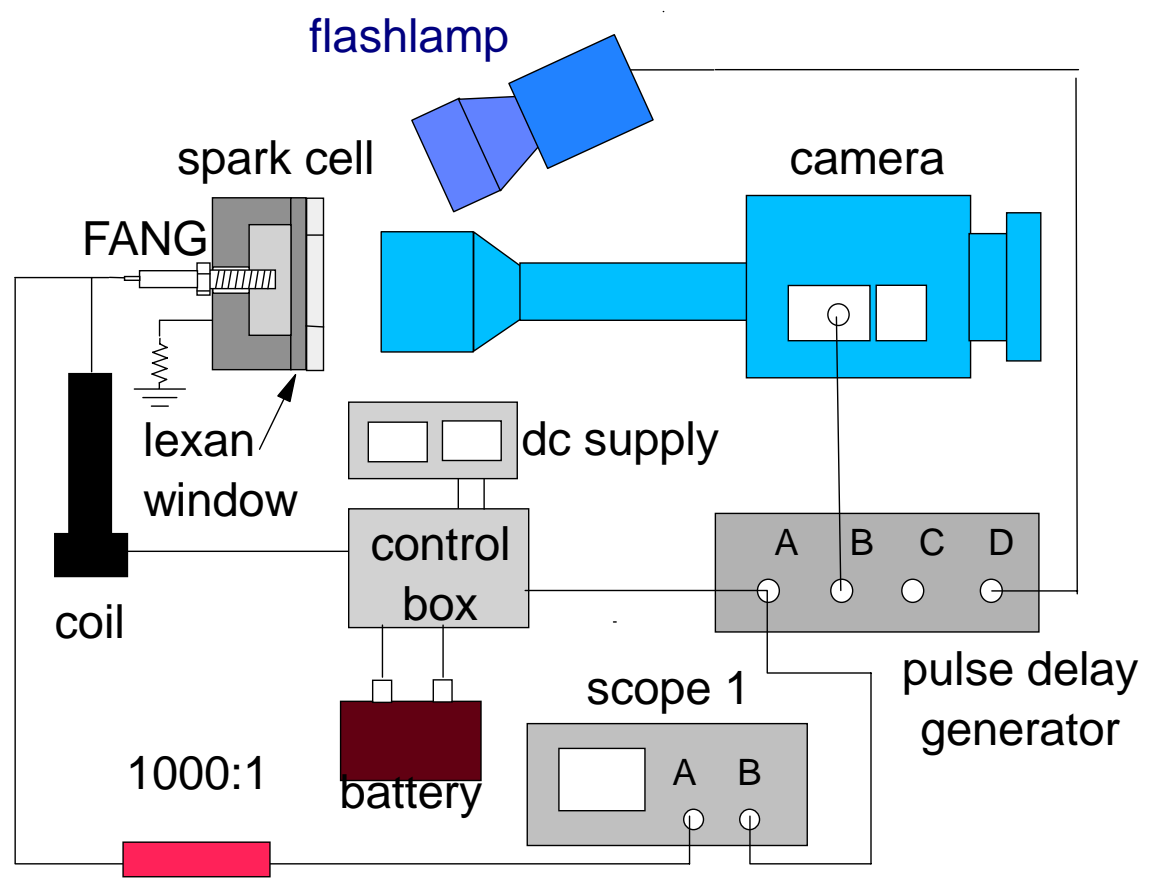

Figure 1. Schematic of experiment.



Figure 2. Photograph of experiment. 


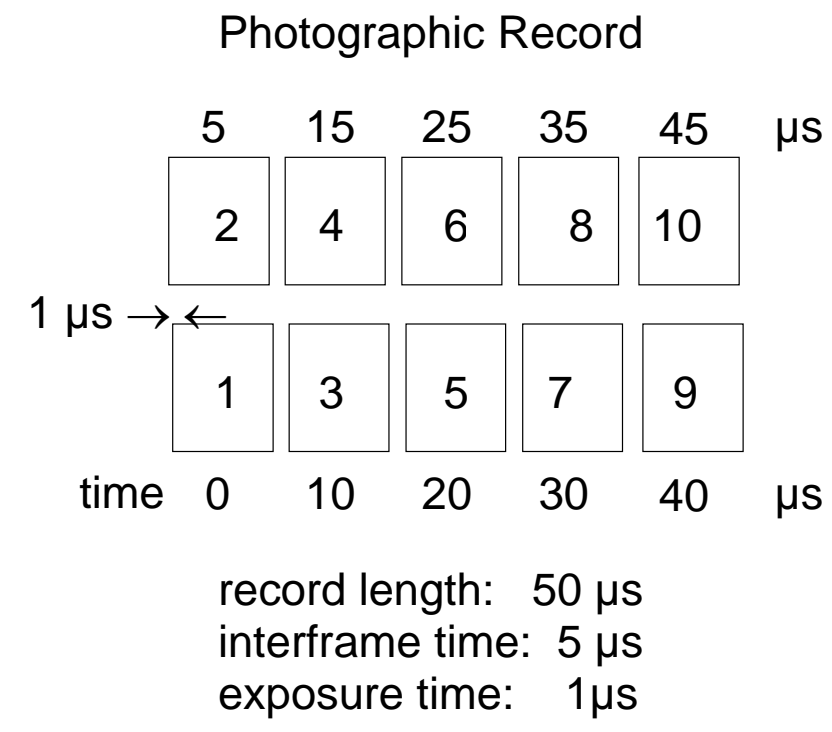

Figure 3. Schematic of photographic record.

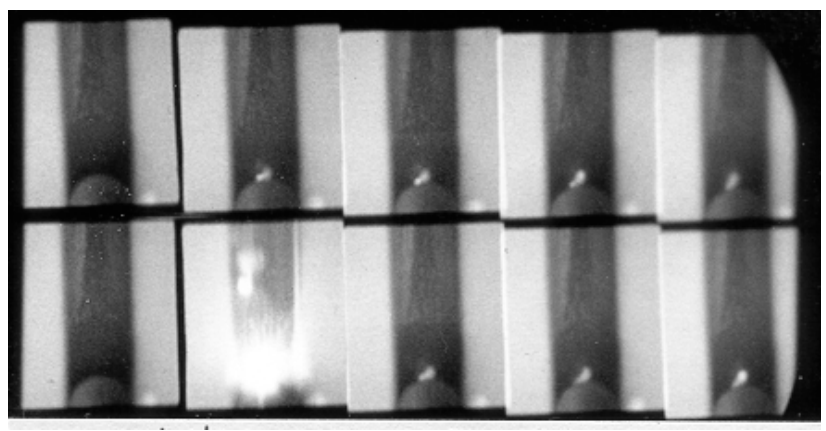

Figure 4. Fast-framing photograph of typical spark event. Initial spark event shown in frame 3. Spark bridges gap and begins movement toward top of plug in subsequent frames. 
arc position, z, vs time ${ }^{1 / 2}$ (discharge along axis)
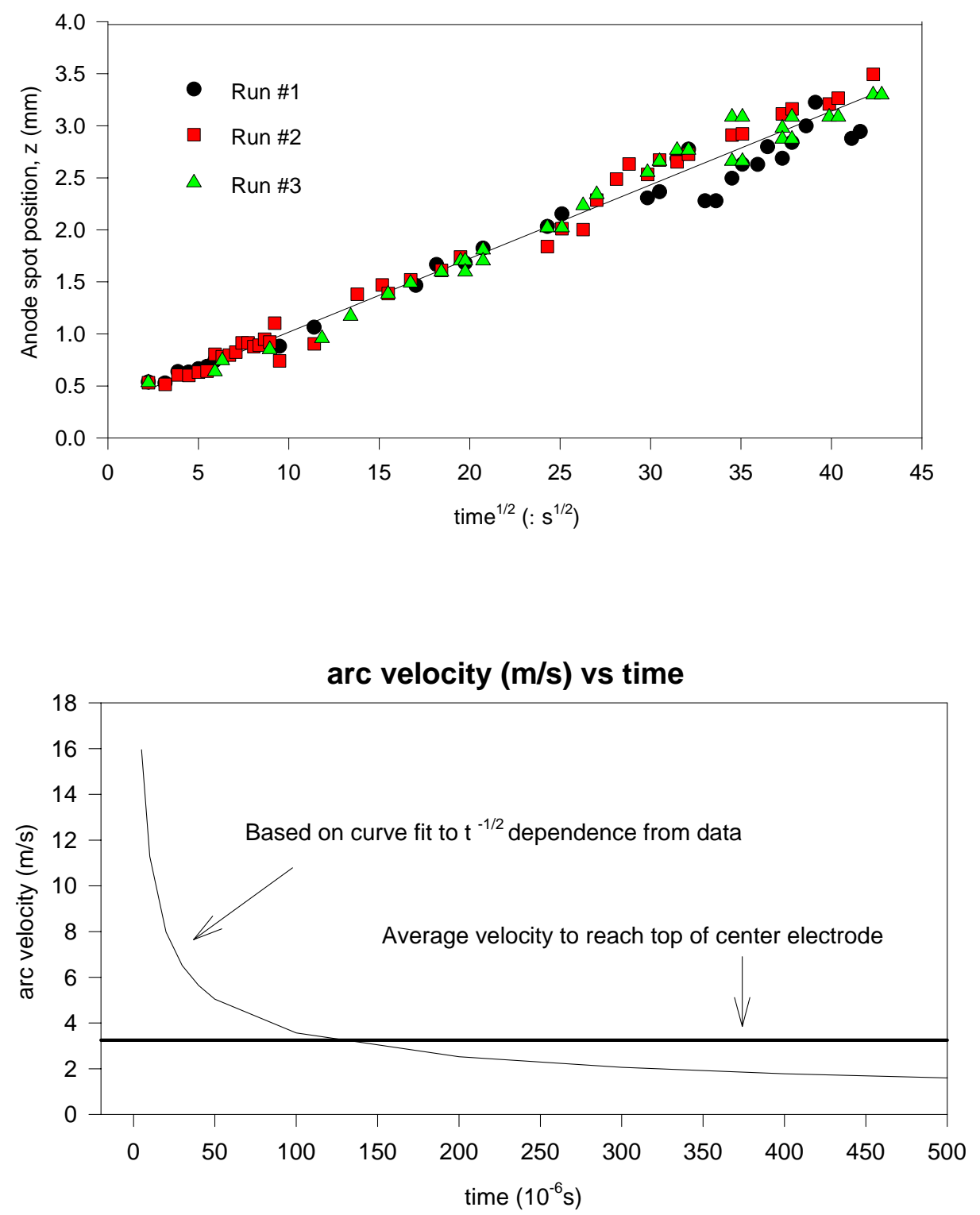

Figure 5. (A) Arc position as a function of the square root of time, (B) Time dependence of arc velocity as derived from arc position data 


\section{APPENDIX A: ARC DYNAMICS MODELS}

Two problems will be discussed. First, the transverse arc velocity in a transverse magnetic field will be considered. An understanding of this matter is important for the proper design and operation of the FANG spark plug. A conventional theory will first be discussed and some of its philosophical and predictive defects will be compared to an alternate theory, which doesn't share these weaknesses. Second, an erosion model is proposed for the FANG spark plug, which explains the laboratory findings. Laboratory tests of the theory are proposed and issues leading to mitigate erosion are proposed.

\section{Arc Velocity}

A conventional formulation of the rod-drag model currently used [3] is

$$
\begin{aligned}
& \frac{d\left(l \pi r^{2} \rho \mathrm{v}\right)}{d t}=J B l \pi r^{2}-C_{d} l \pi r^{2} \rho_{\infty} \mathrm{v}^{2} \\
& \frac{d \mathrm{v}}{d t}=\frac{i B}{\pi r^{2} \rho}-\frac{C_{d} \rho_{\infty} \mathrm{v}^{2}}{\pi r \rho}
\end{aligned}
$$

where Eq (1) is a momentum balance and Eq (2) is the consequent acceleration of the rod (spark channel). In Eq (1), the terms represent the time rate of change of the momentum of the rod, the Lorentz force on a conductor, and the drag force exerted on the rod by the ambient fluid.

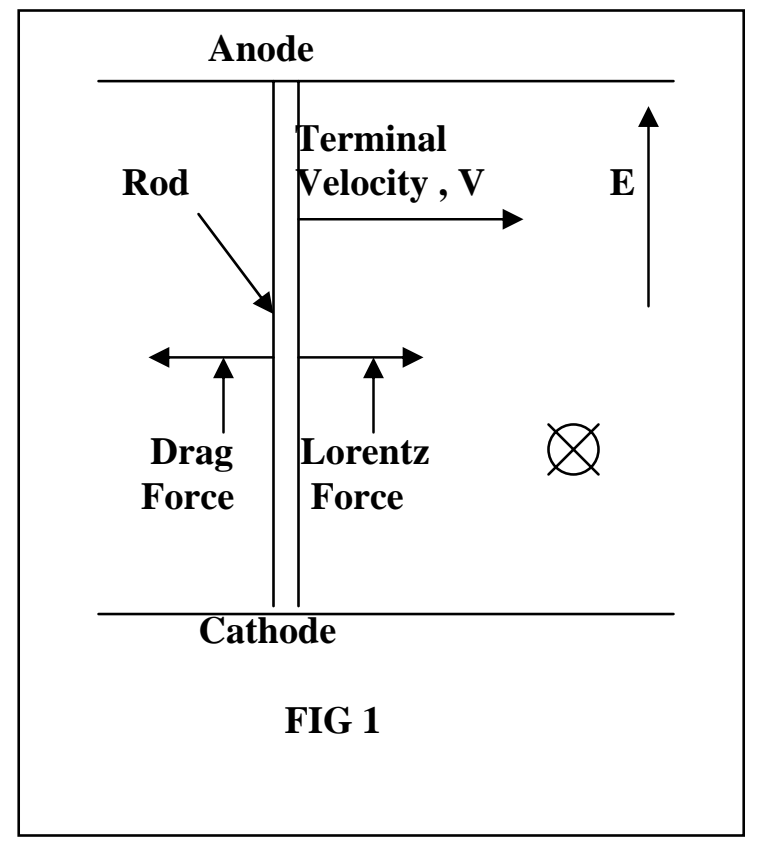

Unfortunately, this model for the motion of arcs in magnetic fields is simplistic, and lacks certain plasma dynamics details.

The problem is that a rigid rod, or current carrying wire, accelerating in a transverse magnetic field is a good model - if there were actually a wire. But in the case of a plasma arc there are several differences: (1) a collisiondominated plasma arc is not a wire, although both can carry current; (2) a collision-dominated plasma can transfer momentum to the neutral gas which escape the confines of the rigid cylinder; this represents a loss term in the momentum balance equations; (3) a plasma entering the arc gap initially takes on the mobility appropriate to the magnetic and electric field, and the momentum transfer cross-section and the local fields, not an initial value related to the exiting charge as is the case for a wire. This represents another alteration to the momentum balance equation; (4) the drag force, for the plasma arc consists of volumetric binary momentum-transfer collisions between the background gas and the ions and electrons in the plasma; for the rod it is a macroscopic force produced of the solid rod by the gas; (5) in the plasma, there are end sheaths in which strong electric fields exist which dominate the charged particle motion-it is not so obvious that this occurs in a rod.

Writing momentum balance equations encompassing items (1-4) is straightforward if we write a microscopic balance instead of a macroscopic balance. Considering that the average momentum communicated by an ion to the gas per collision is [1,2]

$$
\mu \mathrm{v}_{\mathrm{d}}(1-\cos \vartheta)
$$


The total momentum transferred per ion to the gas is

$$
\begin{array}{r}
\mu \mathbf{v}_{\mathrm{d}} \mathrm{N}\left\langle\mathrm{v}_{\mathrm{r}}\right\rangle \int_{0}^{\infty}(1-\cos \vartheta) 2 \pi \mathrm{bdb} \\
=\mathrm{e}(\mathbf{E}+\mathbf{v} \times \mathbf{B})
\end{array}
$$

The ions, instead of reaching a terminal velocity after some macroscopic drag time, as suggested by Eqs. (1) and (2) [4], essentially reach terminal velocity for a momentum transfer relaxation time on the order of 10 picoseconds at 10 atmospheres. This result contrasts sharply with analysis of macroscopic drag formulations, such as Eq. 1 and 2, where terminal velocities are reached in times of the order of microseconds [3].

The theory enumerated in Eq (3) considered only charged particles in a very weakly ionized gas. For sufficient ionization to cause the formation of a plasma with Debye length small compared to the spatial scales of the electrodes, the electric field,

$$
\mathbf{E}=-\nabla \phi
$$

$\mathbf{E}$, is no longer determined from the solution of the "vacuum" potential problem,

$$
\nabla^{2} \phi=0
$$

but needs to include the charge densities. Within the arc plasma, these charge densities are generally large, of opposite sign and almost canceling.

$$
\nabla^{2} \phi=-4 \pi\left(\rho_{+}-\rho_{-}\right)
$$

In a well-established arc, it is at least tentatively plausible that many aspects of the problem can be treated by modelling the positive ions kinetically and the electrons as a Boltzmann distribution.

If the electric field were constant, one could revert to some well-known formulation [5]:

$$
\frac{\partial^{2} \mathrm{n}}{\partial \tau^{2}}+\frac{\partial \mathrm{n}}{\partial \tau}+\frac{\partial \mathrm{n}}{\partial \xi}+\frac{\partial^{2} \mathrm{n}}{\partial \xi^{2}}=0
$$

Green's functions are known analytically for eqn. (7) and are composed of Bessel functions [5], exhibiting shocks and collision-dominated drift and diffusion at the Navier-Stokes level. The transport equation (7) is essentially the same as the diffusion equation except inertial terms are not truncated from the manipulations of the kinetic equations. The interaction force-law between the charged particles and the neutrals and even the fields are incorporated into the spatial and temporal scaling [5]. The gas is assumed to be weakly ionized in the sense that the neutral gas is assumed totally unaffected by the motion of the charged particles.

Dominating over the limited diffusional flow that is implied by Eq (7) are the drifts due to the self-fields of the plasma. This requires a nonlinear description tightly coupling the field equations and the kinetic equations.

Only under a uniform electric field assumption, (and by implication, a very weakly ionized gas) could the arc motion be described as the addition of an electrostatic drift and a magnetostatic drift [7] with some diffusion corrections.

Unfortunately, the electric fields are far from constant, since they are dominated by spacecharge imbalance, especially near the edges of the arc where strong electrostatic sheaths occur. So the kinetic, or transport formulation for the ion density needs to include the fields explicitly (especially those due to space charge), and in fact have to be solved simultaneously with Eq. (6). For example, the positive ion density can be written in terms of the $6 \mathrm{D}$ positive ion distribution function:

$$
\rho_{+}=\int \mathrm{f}(\mathbf{v}) \mathrm{d} \mathbf{v}
$$


A kinetic equation (e.g., the Boltzmann Equation in the binary collision regime) for the $6 \mathrm{D}$ positive ion distribution function is:

$$
\frac{\partial \mathrm{f}}{\partial \tau}+(\mathbf{v} \bullet \nabla) \mathrm{f}+\left(\mathbf{a} \bullet \nabla_{\mathbf{v}}\right) \mathrm{f}=\mathbf{J}(\mathrm{f})
$$

$\mathrm{J}$, in this case, is a binary collision operator, which in some simplifications resembles parts of Eq. (3). The acceleration term in Eq. 9 is just due to the local electric and magnetic fields:

$$
\mathbf{a}=\frac{q}{m}(-\nabla \phi+\mathbf{v} \times \mathbf{B})
$$

Actual explicit individual electrostatic Coulomb interactions are neglected and are realized only as they contribute to the electrostatic potential.

Over most of the interior of the arc plasma, the electron density may be written as a Boltzmann distribution:

$$
\rho_{-}=\exp \left[\frac{-\mathrm{e}\left(\phi-\phi_{0}\right)}{\mathrm{kT}_{\mathrm{e}}}\right]
$$

This is frequently a useful approximation within the arc plasma and at the plasma edge adjacent to negatively biased walls (cathode and sometimes even the anode), when most of the action is on an ion time scale (true for arcs but not for the breakdown phase).

The differences between the macroscopic moving rod approach, (Eqs. 1-2), and a suitably averaged macroscopic approach (Eqs. 4-11) are all treatable at least in some approximate fashion, as will be outlined presently.

The transfer of momentum to the neutral gas can be neglected if this doesn't occur to the extent that the bulk neutral gas flow itself is significantly affected. The same is neglected in the conventional macroscopic treatment. The macroscopic drag force is replaced by the more accurate microscopic drag force arising from binary collisions.

One might wonder if there are some processes occurring within the moving plasma arc that are so different from the rod-drag theory that they are not readily discernable from just looking at the defects of the rod-drag theory. We have proposed a new theory [7], which adds a fundamentally new mechanism for arc drift in a transverse magnetic field. It is as follows. It is normally quite difficult for electrons to advance transverse to the discharge because of electrostatic sheaths that appear tending to confine them to within the arc. In addition, local $\mathbf{E} \times \mathbf{B}$ forces deflect them to the side parallel to the sheath. However, another mechanism whereby electrons can readily get outside of the arc, especially in the advancing direction, starts by secondary electron emission from the cathode due to UV light emission from the plasma and positive ion bombardment down the sheath. Within the essentially 1D cathode sheath, both incoming positive ions and outgoing secondary electrons are deflected in the advancing direction, via simple magnetic drift, Eq (3). In addition, the positive ions are being depleted from the trailing side of the arc with no fresh supply of ionizing electrons.

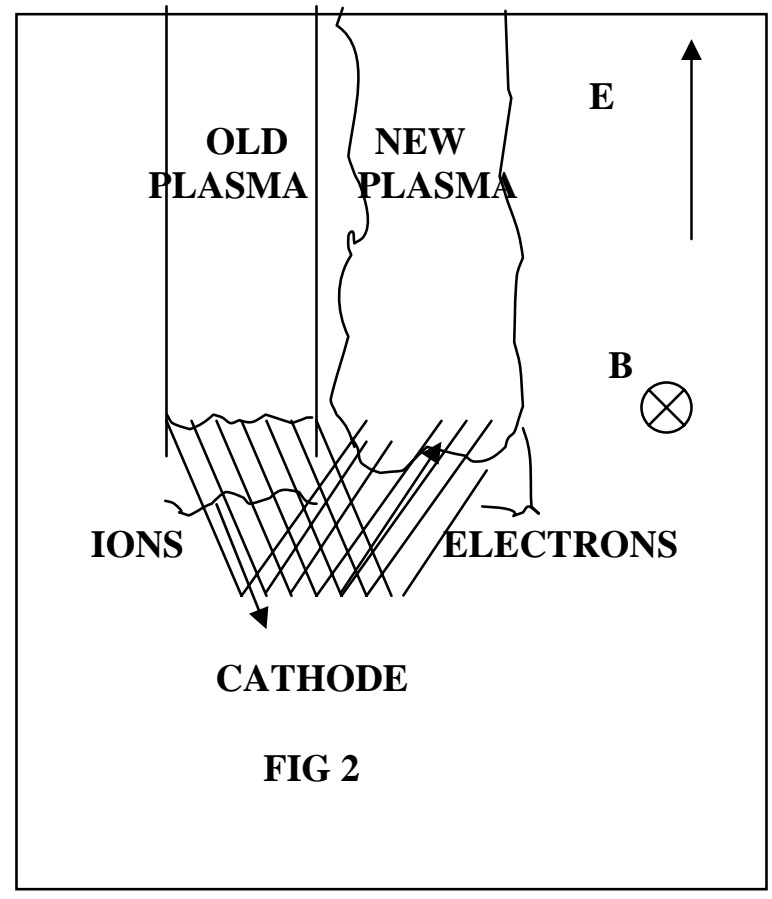


The figure illustrates this mechanism of arc movement. This mechanism of generating more arc plasma in the leading edge and depleting it in the trailing edge gives the appearance of a moving arc. However, the velocity of this moving arc is dependent on other quantities besides that predicted by Eqs (1) and (2). For example, the apparent velocity of the arc depends on how much the positive ions / electrons are displaced during their cathode reflection. If the Debye length of the arc plasma,

$$
\lambda_{\mathrm{D}}=\sqrt{\frac{\varepsilon_{0} \mathrm{KT}}{\mathrm{ne}^{2}}}
$$

is higher, meaning lower plasma density, $n$, and possibly lower gas pressure, $\mathrm{P}_{0}$, then, within limits, the cathode sheath is wider and the electric field lower. In this case, the magnetic forces will cause more deflection and therefore more advance. One may expect that the advance velocity then becomes greater at lower pressure as also predicted by other theories (Eqs. 1 and 2, for example) for somewhat different reasons.

However, the pressure dependence of the transverse arc velocity may be dominated by another effect: the production of secondary electrons from the cathode, which is very strongly dependent on cathode conditions. In particular at high pressure, or high plasma density, the flux of positive ions is greater; both because of their higher initial density and because of the higher drift velocity they have on impact with the cathode. This higher drift velocity occurs since the electric field is larger because the sheath is narrower for the plasma shielding distance of Debye length is shorter. Therefore, the cathode heats up more ejecting more electrons. The higher electric field then carries the electrons to the plasma front quicker and with more collisional ionization producing, more plasma.

What we see in Fig 2 is the extreme case where the plasma leapfrogs an entire arc width per step. For several reasons, this may be unrealistic and the point model would more appropriately described in Fig. 3.

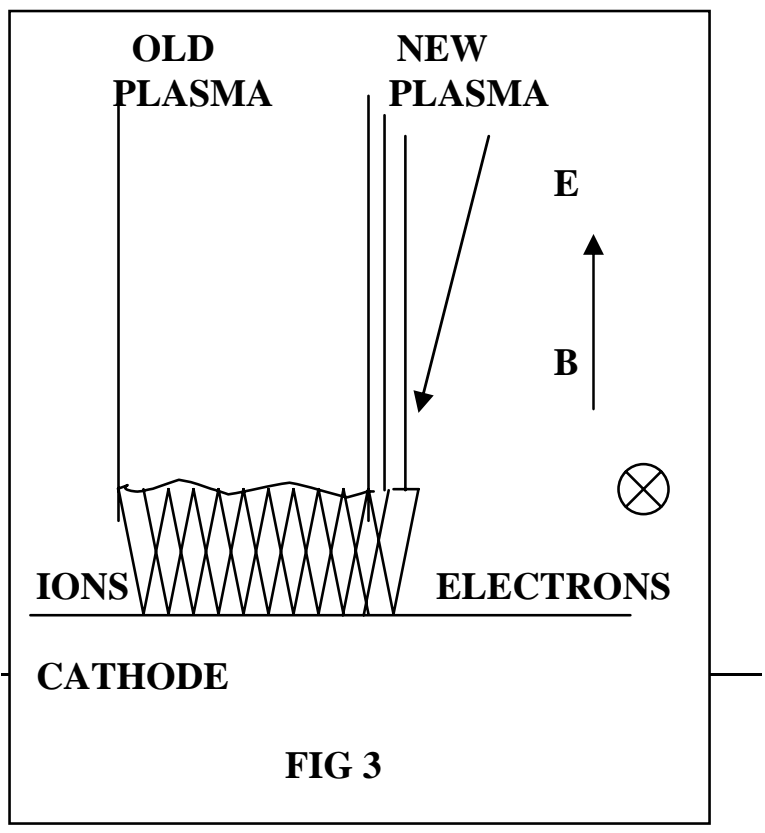

Here the situation is quantitatively different from Fig. 2 in that (1) the new plasma is much narrower than the old plasma; (2) the magnetic drift velocity in the cathode sheath is not as much of a contributor to the total drift velocity; (3) the region of depletion of the old plasma is less so that it lives longer; (4) since the old plasma lingers around longer, it will provide a greater net flux of positive ions to a spot on the cathode enabling more heating of the cathode with more concomitant secondary electron emission; (5) a significant fraction of the electrons propelled into the new plasma can $\mathbf{E} \times \mathbf{B}$ drift along next to the old plasma, getting a rapid transport to the center (towards the anode) and at the same time ionizing the gas. Points 4 and 5 above make the more incremental plasma stepping shown in Fig 3 more robust.

Point 5 above deserves additional explanation since enough detail is not shown in Fig. 3 to explain it. Fig. 3 is expanded and shown in Fig. 4. 


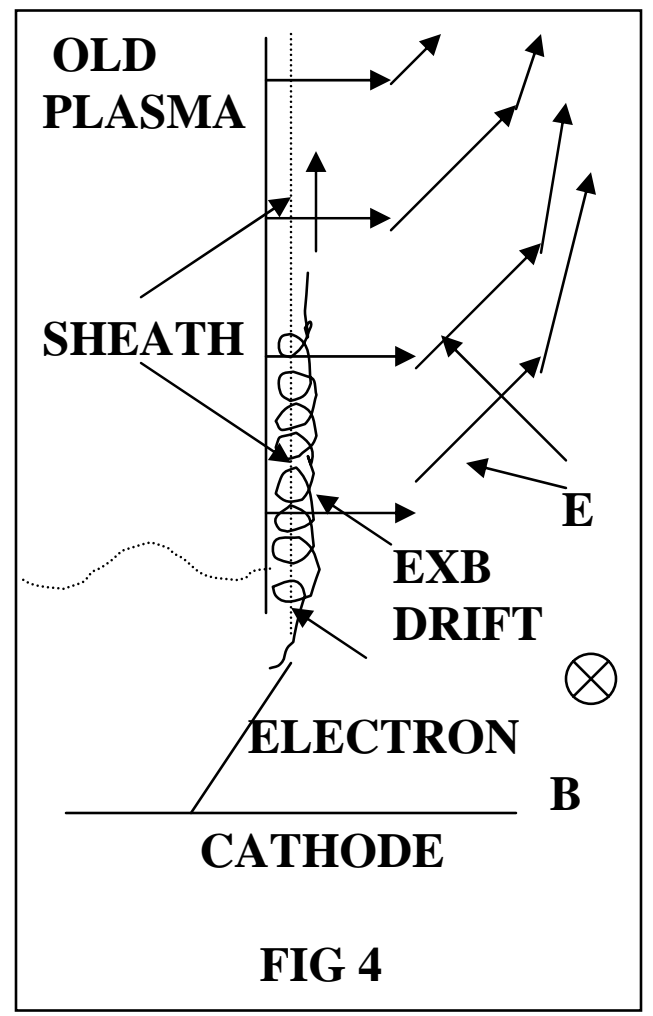

In order to do a tractable calculation it is useful to determine the rate limiting process implied in all the processes occurring in the regions illustrated by Figs. 3 and 4 . The various rate processes include: (1) the transport of positive ions down the "old plasma" to the cathode sheath; (2) the higher speed drift of those positive ions collisionally extracted down the cathode sheath; (3) the formation of secondary electrons on the melting-vaporizing cathode; (4) the fast electron collisional drift up the cathode sheath; (5) the $\mathbf{E} \times \mathbf{B}$ collisional of these electrons along the side of the "old plasma"; (6) the ionization of said electron. These six rates are shown schematically in Fig. 5 using the same representation as Fig. 4. These rate processes will be named as $\mathrm{r} 1$ through $\mathrm{r} 6$ as appropriate in the remainder of this document. Because processes 5 and 6 are not expected to be rate limiting, they will not be discussed here further.

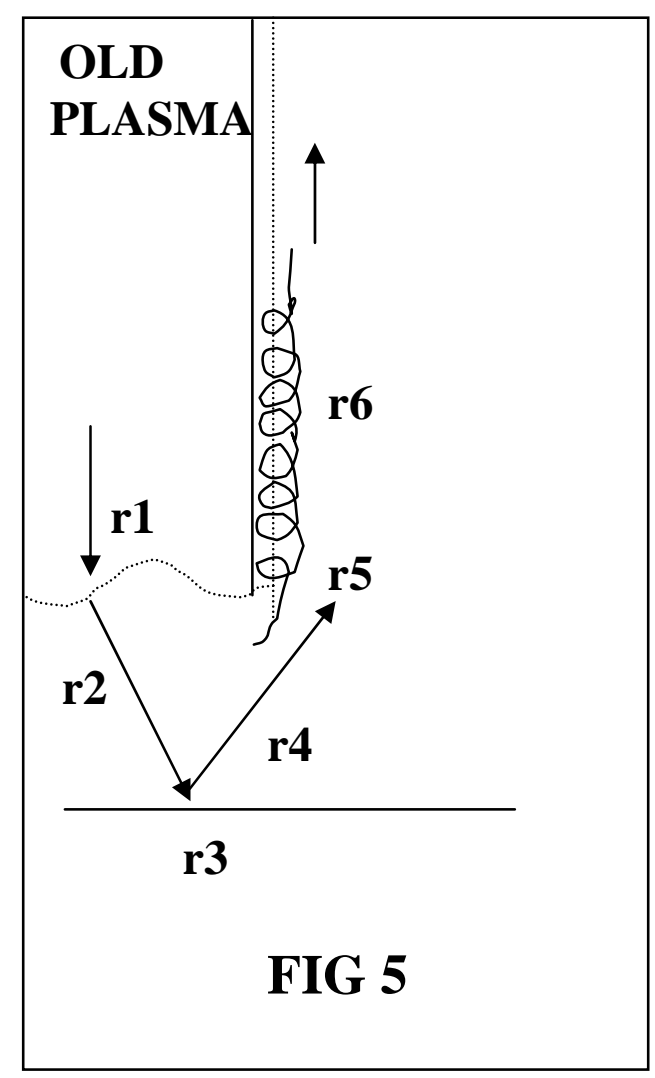

The first item, transport of the positive ions to the cathode sheath is potentially rate limiting since the local electric fields are small. However once the positive ions arrive at the cathode sheath, they are easily extracted, even in the collision dominated regime and so this aspect of the process is not likely to be rate limiting. On the other hand, the formation of secondary electrons at the cathode, from either positive ion bombardment or photon emission from the arc, is a very important link in the generation of the advancing plasma. The secondary electron coefficient is a very strong function of the temperature of the cathode. Below a certain temperature, usually there is no secondary emission at all and above such a threshold, there is an exponentially increasing in secondary production with temperature. It is likely that the parameters, which govern the secondary emission coefficient, dominate the whole rate process. The fast electron drift down the cathode sheath is almost certainly not a limitation. 
First, lets examine the pressure dependence of the plasma evolution velocity. The theory embodied in Eqs. 1 and 2 would predict that the plasma would drift faster at lower pressure (actually inversely proportional to pressure) since the collision frequency is less (actually just Ohms law for charged particle mobility at low fields [2]).

$$
\mathbf{v}_{\mathrm{d}}=\frac{\sigma \mathrm{E}}{\mathrm{P}}
$$

In the macroscopic view, the drag force (see Fig. 1 ) is less at low pressure due to the decreased fluid density. Even in the proposed theory, some of the rate processes appear also to be enhanced at low pressure, particularly r1, r2, and r4. However we will look at the rate processes r1, r2 and r4 more carefully since many other parameters also change when the pressure is changed.

For example in rate process $\mathrm{r} 1$ : transport of the positive ions to the cathode sheath, the collision dominated transport is indeed dependent on $\mathbf{E} / \mathrm{P}$, (as in Eq. 12) indicating at first sight that as $\mathrm{P}$ decreases, $\mathbf{v}_{\mathbf{d}}$ increases. However $\mathbf{E}$ itself, and especially the fluxes, also depends on $\mathrm{P}$. This can be seen most easily in the limit when $\mathrm{P}=0$ where the plasma and plasma shielding vanishes with the vacuum fields. In this case, the electric fields are even higher than the finite plasma case but the flux is zero since no positive ions are generated and thus, no transport. If we consider a more typical 10 atmosphere arc discharge, we find that the flux varies at least directly with the pressure and to a first order approximation the electric field within the plasma is largely independent of pressure. This may change at sufficiently high pressures, or plasma densities because electrostatic instabilities can and do develop [8] which generally lower the rate of positive ion transport. In addition, the distance positive ions have to travel within the plasma is slightly longer with higher pressure due to more extensive plasma shielding, but this is second order since the plasma sheaths are a small fraction of the arc column length anyway. Putting all this together, we can conclude that the largest effect on rate 1 process of a variation of pressure is that:

$$
r_{1}=c_{1} \quad(13)
$$

where $\mathrm{c} 1$ is weakly dependent on pressure. We have assumed that the electric field is unchanged and that the drift velocity is inversely proportional to the pressure.

For rate process r2: the higher speed drift of those positive ions collisionally extracted down the cathode sheath, the collision dominated transport is again dependent on E/P, (as in Eq 12) indicating at first sight that as $P$ decreases, $\mathbf{v}_{\mathbf{d}}$ increases (at constant $\mathbf{E}$ ). However, as before, $\mathbf{E}$ itself, and especially the fluxes, also depends on $\mathrm{P}$. If we consider a more standard 10 atmosphere arc discharge, we find that the flux varies at least directly with the pressure (since this rate 2 (r2) process is in series with the rate 1 (r1) process). However, this time the electric field within the cathode sheath is dependent on pressure since the sheath width is proportional to a Debye length meaning that at higher gas pressure, there is higher plasma density and shorter Debye length. Therefore, since the potential difference, to first order, is the same the field is higher.

$$
E_{2} \propto \frac{\nabla \Phi}{\lambda_{d}} \propto \sqrt{P}
$$

Putting all this together, we can conclude that the largest effect on rate 1 process of a variation of pressure is that:

$$
\mathrm{r}_{2}=\frac{\mathrm{C}_{2} \mathrm{r}_{1}}{\sqrt{\mathrm{P}}}
$$

For rate process r3: the reflection coefficient is a strong function of the temperature of the cathode as previously mentioned. Since the positive ion drift velocity is not expected to be high enough to cause secondary electron emission through an ablation process it is only the temperature of the cathode and how it influences such secondary production processes as photon induced 
emission and positive ion impact emission. The cathode temperature is at least linearly dependent on the arc plasma density $n$, because that determines the incident energy flux.

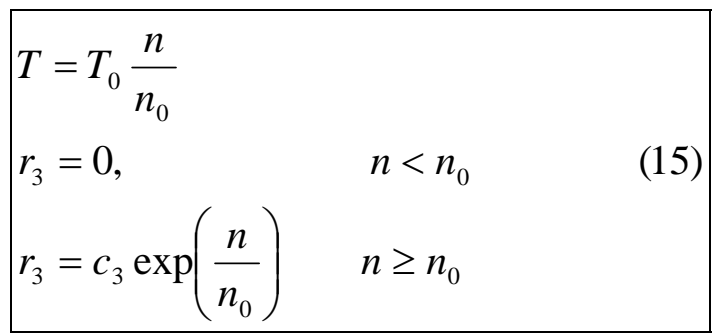

Furthermore, the secondary electron surface production rate $\mathrm{r} 3$ is known to have a threshold temperature, below which no electrons are produced (i.e., glow discharge) and a rapid (greater than linear) dependence on surface temperature above this threshold represented by equation (15). The surface temperature is assumed as proportional to the plasma flux $n$ and the surface production above threshold is exponential as indicated. This rate is probably the most limiting rate of the six rates considered.

For rate process $\mathrm{r} 4$ : the higher speed drift of the secondary electrons emitted from the cathode sheath, the collision dominated transport is again dependent on $\mathbf{E} / \mathrm{P}$, (as in Eq. 12) indicating at first sight that as $\mathrm{P}$ decreases, $\mathbf{v}_{\mathbf{d}}$ increases. However, as before, $\mathbf{E}$ itself, and especially the secondary emission coefficient, also depends on P. If we consider a more typical 10-atmosphere arc discharge, the electric field within the cathode sheath is dependent on pressure in the same way as r2.

Putting all this together, we can conclude that the largest effect on rate 1 process of a variation of pressure is that:

$$
r_{4}=\frac{c_{4} r_{1} r_{2} r_{3}}{\sqrt{\mathrm{P}}}
$$

Now to compute the velocity of the transverse arc motion from these considerations, we need only stipulate that

$$
\begin{array}{rlrl}
\mathrm{v}_{\perp} & =r_{4} & \\
& =0, & & n<n_{0} \\
& =\frac{c^{\prime}}{P} \exp \left(\frac{n}{n_{0}}\right) & & n \geq n_{0}
\end{array}
$$

Now this theory, in a general way adds to the result of the theory of Eqs. 1 and 2 by the addition of plasma dynamic effects.

The experimental results of arc transverse velocity vs pressure is as follows [9].



Theory 1 refers to Equations 1 and 2 (the roddrag theory); Theory 2 refers to $\mathrm{Eq} 17$ (the secondary production theory). Note that the secondary production theory, as represented, predicts a threshold at a certain pressure, $\mathrm{P}_{0}$, whereas the rod-drag theory predicts that the transverse arc velocity will get larger and larger (without bound) as the pressure get smaller. Clearly, from the data points represented in Fig. 6 , the secondary production theory is at least qualitatively correct at low pressures and the rod-drag theory is not correct. The threshold behavior of the transverse arc velocity at low pressure is a direct result of our conjecture on the threshold behavior of the secondary emission coefficient of the cathode as a function of temperature. It would be more reasonable to assume a somewhat more gradual onset of 
secondary electron emission than that of a sharp threshold. Such an assumption would bring the theory yet more into agreement with the laboratory data. In order to assess whether the apparent disagreement with the penultimate data point shown in Figure 6, we would need to know more about the measurement uncertainties.

\section{Erosion in FANG plug}

Dominant erosion processes involve the interaction of high energy (compared with the gas) charged particles with the electrodes. There are three distinct phases: electrons striking the anode just after breakdown, and electrons and positive and electrons striking the cathode and anode (respectively) during the arc phase. The breakdown phase lasts only a nanosecond or so one might be tempted to dismiss this source of erosion compared to the processes occurring during the arc phase which can last for as long as a million times longer. However, the electrons during the breakdown phase have much higher density and energy then in the arc phase. In addition the events occur so rapidly in the breakdown phase that thermal conduction within the electrodes does not have a chance to act. Therefore the anode can be made to efficiently boil off material. Regular spark plugs are susceptible to all three types of the above mentioned erosion mechanisms. The FANG plug is much less susceptible to the arc erosion processes since the arc is moving and covers a relatively large area. However it is still and perhaps equally susceptible, compared with conventional spark plugs, to the breakdown erosion mechanism since no appreciable arc motion occurs during this time scale.

The following erosion model for the FANG plug is therefore proposed.

First we note that "slanted," or ground, electrode is the anode and attracts electrons, we could identify that electrons are doing the eroding, presumably by local vaporization (since unlike ions, sputtering-like phenomena is not likely due to a dearth of momentum). According to a breakdown-to-arc transition model [10], the likely occurrence of this erosion is at the end of the breakdown phase where an outburst of electrons strikes a small area on the anode (slanted, ground) electrode. This outburst occurs at relatively high energy (at least several $\mathrm{eV}$ ) and high current (tens of amperes, but for only a nanosecond).

According to this model, the electron beam vaporizes a little of the anode electrode. On the next shot, the electron beam vaporizes another spot. Finally, the erosion is like an electron beam milling machine, rastering up the unslanted section of the anode and then starting up from the bottom again, repeating the process. After this, the unslanted part of the anode results in an eroded shiny mirror-like surface.

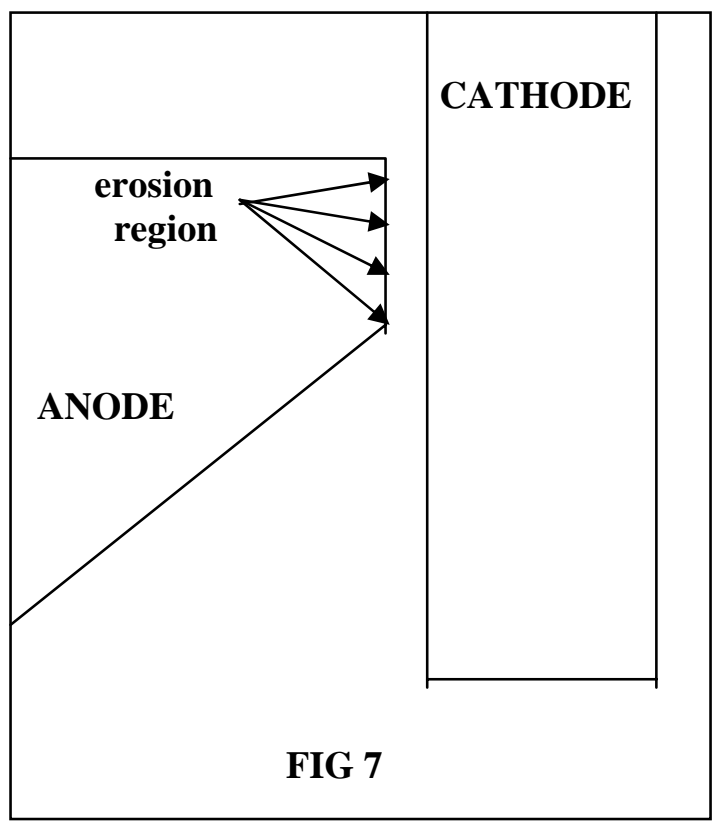

This mechanism explains why the anode electrode in the FANG plug becomes significantly more eroded than the cathode, and why the slanted part of the FANG gets much less erosion (the breakdown only occurs more inside the plug than the slant location as shown in Fig. 7).

For a regular spark plug, by contrast, the cathode also is eroded because of positive ion impact, which occurs in the same place throughout the length of the arc. For the FANG plug, the 
positive ion erosion is diluted over a much larger area due to the arc movement in the magnetic field. The slanted part of the anode gets much less erosion, compared to the straight part, because the end of the breakdown phase, where the erosion occurs, only happens at the beginning of the discharge which in turn only happens at the straight part of the anode.

Reversing the polarity of the spark plug electrodes can make a sufficient laboratory test of this mechanism. If the proposed mechanism is dominant, then the center electrode will be eroded only at the part opposite the flat part of the slanted electrode. If this happens, it can be argued that most other erosion mechanisms are less important than the proposed one because other erosion patterns would happen.

If the proposed electron "spike" at the end of breakdown mechanism is dominant, then a reduction in erosion can be obtained by reducing the peak current during the end of the breakdown phase. A series resistor comes to mind but this would reduce the current, lest we reduce the ignitability of the plug. Therefore we need a "smart" resistor, or current limiter, to reduce the current only during the avalanche outburst and not later (or when the current is greater than a certain amount). Another method is to use a material on this part of the anode, which has a high vaporization point, but retains a large secondary electron reflection coefficient.

\section{References}

[1] R.D. Present, Kinetic Theory of Gases, McGraw-Hill, (1958), p.136.

[2] McDaniel and Mason, The Mobility and Diffusion of Ions in Gases, Wiley, 1973, p133.

[3] J. Conklin, internal communication (1999).

[4] Actually, Eqs 1 and 2 are a macroscopic momentum balance for a rigid rod in an infinite fluid. The solution of the equations requires an explicit initial condition. For the steady-state solution depicted in Fig. 6 as Theory 1, the transient terms are set to zero. Such a steadystate treatment was carried out in [3].

[5] J. H. Whealton, Phys. Rev. A11, p. 297 (1975).

[6] D. D. Paul, private communication (1999).

[7] Suggested at discussions at Cummins Oct 25 (1999).

[8] J. H. Whealton, M. A. Bell, R. J. Raridon, K. E. Rothe, and P. M. Ryan, J. Appl. Phys. 64 6210 (1988).

[9] I Sauers at Cummins discussions on Oct 25, (1999).

[10] Whealton proposal at Cummins, Oct 25 (1999). 